\title{
Diseño mediterráneo. Análisis preliminar de epígrafes.
}

\section{Picca, Laura.}

PhD candidate - Universitat Politècnica de València, Spain - laurapicca@hotmail.com

\begin{abstract}
Resumen
Este artículo trata sobre el Diseño Mediterráneo como elemento de identidad cultural y tiene como objetivo fundamental identificar a través de la revisión literaria aquellos epígrafes que definen a la "identidad mediterránea" en el área del diseño industrial. Grandes diseñadores y profesionales emergentes se cuestionan y preguntan sobre la continuidad de la profesión en estos tiempos de crisis, por lo que se hace necesario comprender que el diseño no es ajeno a los cambios sociales, económicos y políticos, que la cultura del proyecto ha de ampliarse para generar alternativas más sensibles con la realidad. Frente a lo ampliamente hablado del "estilo mediterráneo" desde diferentes disciplinas como la arquitectura, la moda, incluso la cocina con la denominada "dieta mediterránea", es que este análisis preliminar tiene como objetivo centrarse en la naturaleza que define la "mediterraneidad". Todo ello atendiendo a las variables socio-culturales, económicas y políticas en proyectos y acciones como el Corredor Mediterráneo, el cual define el marco geográfico de actuación (el Arco Mediterráneo Latino). Se detecta en primer término; la existencia de empresas que actualmente y a modo intuitivo utilizan la "identidad mediterránea" en el diseño y desarrollo de sus productos; y en segundo lugar un importante incremento de los valores de autenticidad, respeto por el medio y los productos naturales, junto al valor cultural asociado al producto. Conviviendo los binomios artesanía-producción industrial y los regionalismos en un mercado globalizado.
\end{abstract}

Palabras Clave: Diseño, Mediterráneo, Identidad. 


\begin{abstract}
.
This article deals with the Mediterranean Design as an element of cultural identity and its main objective is to identify through the literary revision those epigraphs that define the "Mediterranean identity" in the area of industrial design.

Great designers and emerging professionals question and ask about the continuity of the profession in these times of crisis, so it is necessary to understand that design is not alien to the social, economic and political changes, that the culture of the project has expand to generate alternatives more sensitive to reality.

Faced with the widely spoken "Mediterranean style" from different disciplines such as architecture, fashion, even cooking with the so-called "Mediterranean diet", is that this preliminary analysis aims to focus on the nature that defines the "Mediterranean"

All this taking into account socio-cultural, economic and political variables in projects and actions such as the Mediterranean Corridor, which defines the geographical framework of action (the Latin Mediterranean Arc).

It is detected in the first place; the existence of companies that currently and intuitively use the "Mediterranean identity" in the design and development of their products; and second, an important increase in the values of authenticity, respect for the environment and natural products, together with the cultural value associated with the product. Living together the handicrafts-industrial production binomials and regionalisms in a globalized market.
\end{abstract}

Keywords: Design, Mediterraneo, Identity.

\title{
1. Introducción
}

"Se conoce como carácter mediterráneo; distendido, amigable, familiar... y para los disciplinados, tremendamente exasperante...

...Se tiene, al ser mediterráneo, por alguien que sabe vivir la vida, sin por ello dejar de sacar adelante sus objetivos, pero excesivamente permisivo con los fallos, los errores, las 
imprecisiones. Además de ser muy hábil improvisando para sortear obstáculos y contratiempos." Mundet (2009) ${ }^{1}$

\section{Definición de términos.}

\subsection{Diseño}

El diccionario de la R.A.E 2014 define

Diseño; (Del it. Disegno).

1. m. Traza o delineación de un edificio o de una figura.

2. m. Proyecto, plan. Diseño urbanístico.

3. m. Concepción original de un objeto u obra destinados a la producción en serie.

Diseño Gráfico, moda, industrial.

4. m. Forma de cada uno de estos objetos.

5. m. Descripción o bosquejo verbal de algo.

6. m. Disposición de manchas, colores o dibujos que caracterizan exteriormente a diversos animales y plantas.

En estas acepciones se pone de manifiesto la dificultad de definir el término; por un lado se refiere a una actividad: concepción de un objeto, producción en serie y por otro se refiere al resultado de dicha actividad; dibujo, maqueta, producto.

Margolin y Buchanan (1995) sostienen que el primero en referirse al diseño como término, fue Aristóteles, definiéndolo como "la previsión de las consideraciones específicas de cada tipo de tarea".

Sparke (2011) dice que el concepto diseño es amplio y complejo ya que tiene su raíz en la palabra italiana Disegno y la francesa Dessin, pudiendo utilizarse tanto como verbo to design diseñar como sustantivo design diseño.

En la traducción castellana del término Industrial design, de los países de lengua anglosajona Design, (del latín designare: delimitar, trazar, indicar), se acentúa el significado proyectual del término.

\subsection{Diseño industrial}

En su definición primigenia al diseño industrial se lo definía como la proyectación de objetos fabricados industrialmente.

\footnotetext{
${ }^{1}$ Mundet, J. de formación continua d' ESCI.
} 
El ICSID, consciente de la evolución y los cambios de la sociedad en que nos encontramos hoy, modificó en 2005 su definición de diseño industrial de acuerdo a una sociedad inmaterial, como lo es la sociedad de principios de siglo XXI:

"El, diseño es una actividad creativa, cuyas directrices establece múltiples facetas y cualidades de los objetos, procesos, servicios y sistemas a lo largo de todos sus ciclos de vida. Por lo tanto, el diseño es un factor primordial de la innovación humana, de las tecnologías y un factor crucial del intercambio cultural y económico" ${ }^{2}$

El autor Margolin (2009) propone que:

"el diseño es el poder humano de concebir, planificar y crear productos que sirvan a los seres humanos para la consecución de sus propósitos individuales y colectivos"..."los diseñadores están continuamente inventando nuevos contenidos, por consiguiente, es imposible limitar la investigación del diseño a un conjunto fijo de productos materiales o inmateriales."

Definición que claramente refiere al compromiso social y ético de la profesión del diseñador industrial.

Triana et. al. (2013) en su tesis doctoral cita a Michael Enhoff (2002) que concluye en lo siguiente:

"El diseño al contrario que el arte, necesita de un fin práctico y lo encuentra en cuatro requisitos: ser funcional, significativo, concreto y tener una aplicación social" DZ Centro de Diseño (2002).

Autores y teóricos del diseño como Bonsiepe (1985), Margolin (2005) (2006), Ricard (1982), Maldonado (1990), Manzini \& Jegou (2003), se manifiestan contrarios al accionar de diseñadores que atienden ante todo a los intereses industriales, escasos de reflexión y teniendo como consecuencia la alta producción material y la desigualdad social. Insistiendo en la necesidad de vincular la actividad del diseñador industrial con la sostenibilidad ambiental, el respeto y el equilibrio social.

Poynor (2012) dice:

"Al final de las décadas de los ochenta el término diseño utilizado como prefijo, casi se transformó en un término abusivo. Por una parte sugirió superficialidad y vacuidad y por otra la inútil reformulación de las cosas más comunes (agua mineral de diseño) y en el peor de los casos -drogas de diseño-, el prefijo fantaseaba un mundo de superficies de glamour que escondía una subyacente amoralidad, falta de afecto y hasta corrupción.”

\footnotetext{
${ }^{2}$ ICSID (Consulta junio 2012). Definition of design [on line]. Montreal, Québec.

En $<$ http://www.icsid.org/about/articles31.htm>
} 
Guy Julier (2006) remarca la importancia del papel del diseño en la creación de identidades, hábitos de consumo y estilos de vida, concibiendo el diseño como una práctica cultural que tiene un valor económico.

"El diseño no puede ser entendido desde un punto de vista individualista, aislado, sino como el resultado de movimientos nacionales e internacionales, con sus flujos e identidades".

El diseño se mueve en múltiples escenarios;

- $\quad$ es acción (se desarrolla a partir de metodologías y procesos)

- $\quad$ es sujeto (mediante la concreción de productos)

- $\quad$ es herramienta (es un medio de colaboración entre diferentes disciplinas)

- $\quad$ es adjetivo (“de diseño”, mediatizado por diseñadores famosos o de Marca). (Fig. 1)

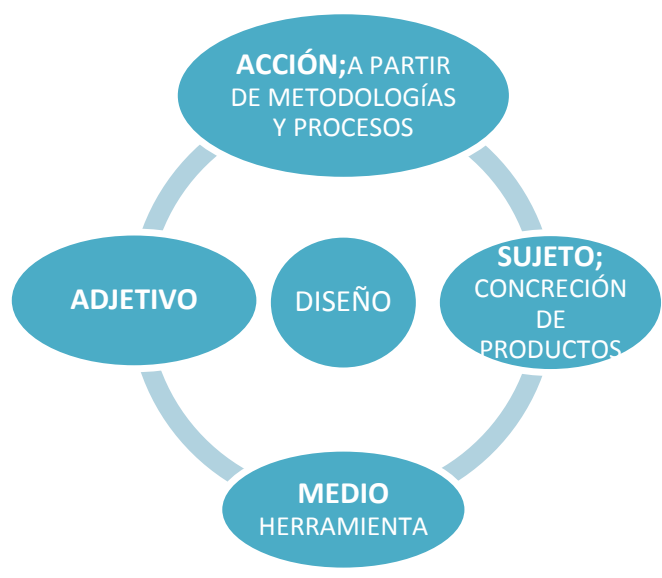

Fig. 1. El diseño y sus escenarios.

La dificultad de encontrar una definición de diseño universalmente aceptada, radica en que cada definición se asocia al momento histórico o incluso a la especialidad en que se desenvuelve el teórico o autor que lo define.

Todas estas actividades o profesiones deberían trabajar juntas, como equipo interdisciplinar integrador; por una mejora global de la calidad de vida. Por eso, el termino diseñador se refiere al individuo que ejerce una profesión intelectual y no simplemente un trabajo formal o de servicio para una marca o una empresa. Fig. 2. 


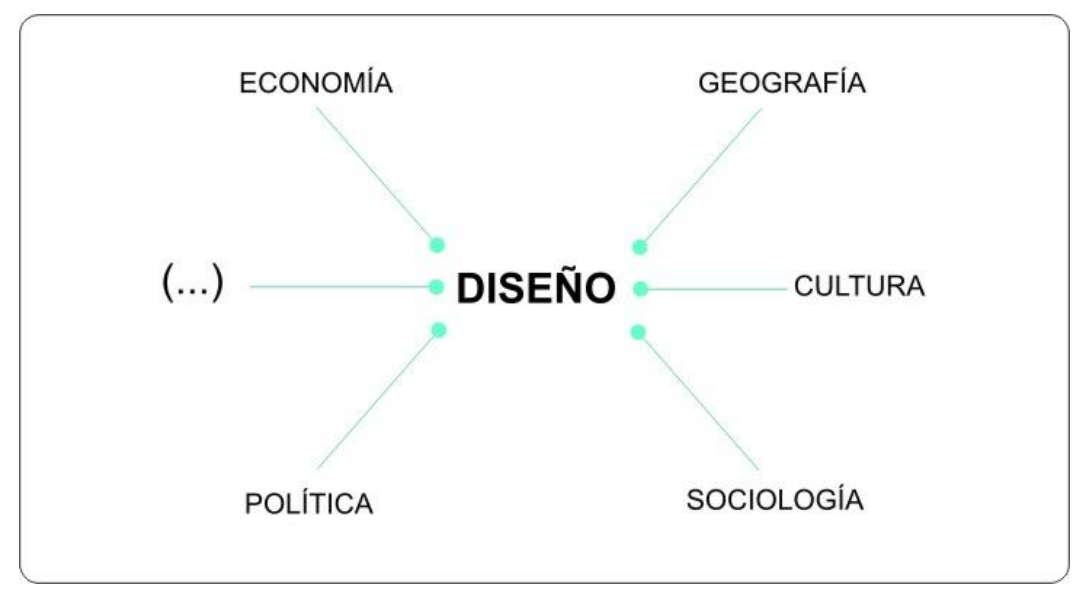

Fig.2 Interrelaciones del Diseño con otras disciplinas.

Esta definición atribuye al diseño conceptos como ética global, ética social y ética cultural, que deben mejorar el valor de la vida, ampliando así el concepto de diseño vinculado a la necesidad material.

En esta misma línea Norberto Chaves (2012) define el diseño del siguiente modo:

"EI diseño es la disciplina que se encarga de dotar de valor simbólico explícito al producto industrial; es la cultura de la industria. Para el diseño cualquier condicionamiento utilitario o tecnológico constituye la base significante de una propuesta simbólica".

\subsection{Cultura del diseño.}

Según (Julier, 2010) una identidad está marcada por todas las circunstancias que influyen en esa materia, viéndose el diseño influenciado por la geografía, la cultura, la política, la economía, etc.

Así, distingue Julier los tres momentos históricos en la creación de identidades nacionales en el diseño: Fig.3. 
Comienzos del S.XX.

Búsqueda de las características nacionales de Diseño en el escenario Internacional del Art Nouveau.

Fin de $2^{\mathrm{a}}$ G.M.:

Reconstrucción de los Estados Nación. Búsqueda de lo cotidiano, lo reconocido y las prácticas locales.

S. $X X$

1990. Contexto de reivindicación, intención de expresar la pureza y naturalidad de lo local. Transmisión de tradición, herencias y costumbres.

Fig.3. Evolución y etapas de creación de identidades, según Julier (2010).

1. El rol del diseñador en la creación de "valor".

2. Circulación. Movimiento del diseño alrededor de los procesos productivos, tecnologías disponibles, factores económicos y políticos.

3. La práctica. Los individuos como hacedores de prácticas colectivas.

\section{(Fig.4)}

Finalmente el término "cultura del diseño" propuesto por Julier resalta la importancia de que los productos se comuniquen con los usuarios, transmitiendo una identidad, por lo que ya no solo es necesario que existan una serie de valores, sino que es "vital" que estos sean detectados por los receptores: los usuarios (Chaves, 2006). 


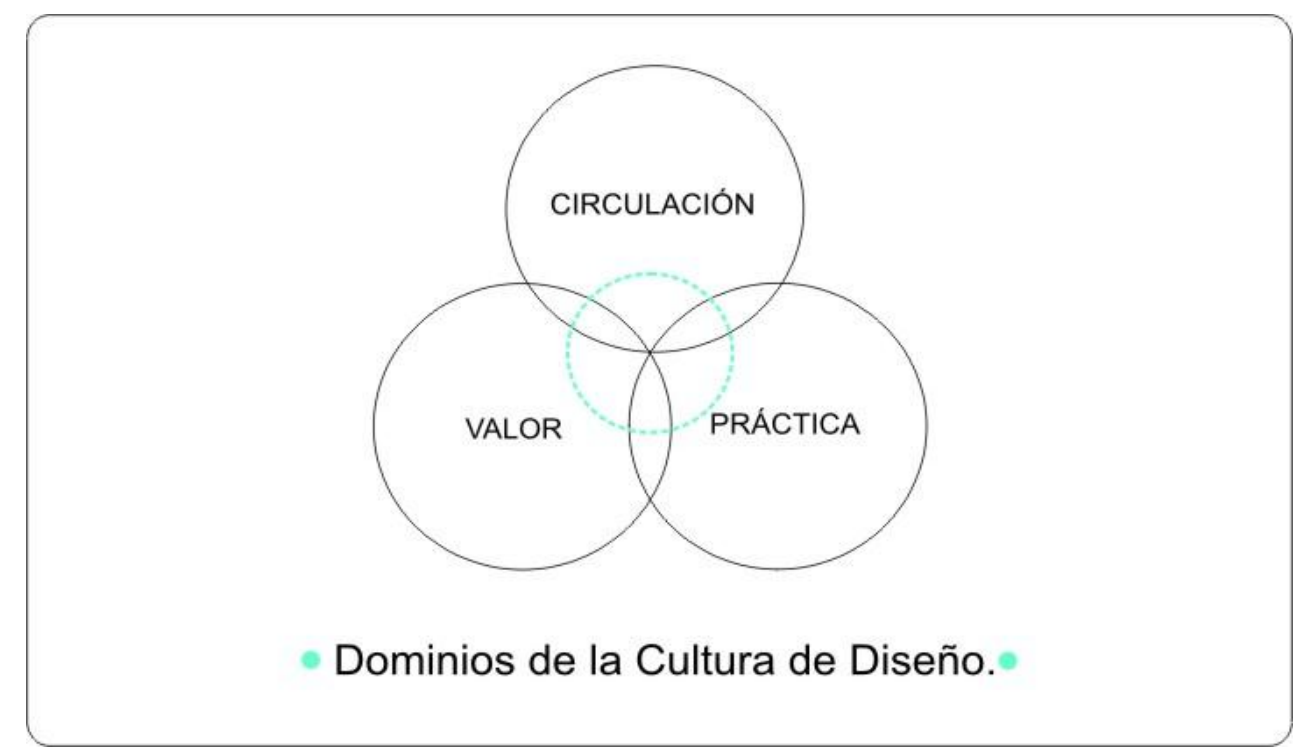

Fig.4. Dominios de la cultura del diseño.

En cambio, destaca por encima de todos estos valores, la importancia de la pertenencia a una determinada identidad como agente o motivo de consumo. Un claro ejemplo es el diseño escandinavo y la explotación cultural y económica de su identidad.

\subsection{Identidad.}

El concepto de identidad extraído del diccionario de la R.A.E. ed. 2014:

Identidad (Del b. lat. identitas, -atis).

1. f. Cualidad de idéntico.

2. f. Conjunto de rasgos propios de un individuo o de una colectividad que los caracterizan frente a los demás.

3. f. Conciencia que una persona tiene de ser ella misma y distinta a las demás.

4. f. Hecho de ser alguien o algo el mismo que se supone o se busca.

5. f. Mat. Igualdad algebraica que se verifica siempre, cualquiera que sea el valor de sus variables.

Según Molano (2008), el significado de identidad se aleja del concepto de individualidad y se refiere a una cualidad o conjunto de cualidades con las que una persona o grupo de personas se ven íntimamente conectados. En este sentido la identidad tiene que ver con la 
manera en que individuos y grupos se definen a sí mismos al querer relacionarse "identificarse"- con ciertas características. Es un término complejo ya que posee un carácter cambiante que funciona individual y colectivamente influenciado constantemente por el exterior. $^{3}$

Las primeras teorías sobre la identidad hablan de este término como el Yo, remarcando la auto-identificación o identidad personal (Cooley, 1922).

Si la identidad no es una esencia innata dada, sino un proceso social de construcción, se requiere establecer los tres elementos constitutivos a partir de los cuales se construye (James, 1890).

\subsection{Identidad Mediterránea}

El Mediterráneo fue el primer mar nombrado y localizado, el primero en conquistar su individualidad toponímica. Desde un principio se lo califica de mar entre tierras, así que de manera singular reúne dos oposiciones; tierra y mar.

Por lo que la cuenca mediterránea explota una concepción de la sociedad basada en el individuo, en la familia, en la producción y en el intercambio.

\subsubsection{Escenario geográfico. Arco mediterráneo.}

Las regiones del Mediterráneo Occidental o Arco Latino, que comienza en Andalucía y se extiende hasta el Lazio, suponen un conjunto dotado de potencialidades de integración propia. Sólo en su dimensión territorial, constituyen el 14 del territorio de la Unión Europea con una población global de 40 millones de habitantes, alcanza el 12\% de la población comunitaria. $^{4}$

Según Rituerto (2010) que afirma; que en la Europa mediterránea se describen espacios socioeconómicos que a pesar de sus niveles de desarrollo desigual, engloban regiones con intereses y características comunes. ${ }^{5}$

Su desafío para los próximos años es la conformación de un eje económico mediterráneo que contribuya a cohesionar el crecimiento de la Unión, comprometiendo, a su vez, a ésta con el desarrollo de los países ribereños no comunitarios. Variables socio-culturales, económicas y políticas con proyectos y acciones como el Corredor Mediterráneo, como apuesta de integración económica.

Esta franja es la zona de mayor peso económico y desarrollo industrial del Mediterráneo,

\footnotetext{
${ }^{3}$ Molano, O, L. (2008). Identidad cultural: un concepto que evoluciona. Revista Opera, No7, 69-84.

${ }^{4}$ Tendencias interculturales euro mediterráneas 2010. Fuente: http://www.esci.es/escivista/num8/articles/04.htm

${ }^{5}$ Martínez de Rituerto, R. Bruselas. SEP 2010
} 
(también denominado Arco Mediterráneo o Mediterráneo Latino) ${ }^{6}$

\subsubsection{Consideraciones socio-culturales del mediterráneo latino.}

"Siesta, familia, relax... el estilo de vida mediterráneo vuelve a estar de moda" ${ }^{7}$ Tendencias interculturales mediterráneas (2010)

Aunque se habla de la "cultura europea" haciendo referencia a una historia, unas tradiciones, una forma de actuar y de pensar propia de este continente, en Europa también existe una diversidad muy amplia de culturas y subculturas.

La mayor distinción la podemos centrar entre la cultura mediterránea, propia de España, Italia, Francia o Grecia y una cultura anglosajona, característica de países más septentrionales como el Reino Unido, Alemania, Holanda o los países escandinavos. ${ }^{8}$

En el norte de Francia, con frecuencia se pueden apreciar patrones de comportamiento más propios de la cultura anglosajona, al igual que en el norte de Italia. En España, podemos distinguir también diferentes culturas o subculturas: una cultura mediterránea en Cataluña o Valencia; una cultura vasca, etc.

"Las empresas que operan en Europa coinciden en que una de las principales dificultades a la hora de negociar acuerdos la constituye las diferencias culturales".

Existen una serie de valores y dimensiones propias de la cultura mediterránea o del Sur de Europa y unos valores y dimensiones características de la cultura anglosajona y del Norte de Europa.

\subsection{Diseño e identidad}

"Ha habido una verdadera explosión teórica en los años recientes en torno al concepto de $<$ identidad $>$. Actualmente no hay al parecer ningún otro aspecto de la vida contemporánea que atraiga en la misma medida la atención de filósofos, científicos sociales y psicólogos. (...), comenta que $<<$ estos estudios sobre la identidad $>>$ se están convirtiendo con gran celeridad en una próspera industria; y más aún, se puede decir que la $<$ identidad $>$ se ha convertido ahora en un prisma a través del cual se descubren, comprenden y examinan todos los demás aspectos de interés de la vida contemporánea. (Bauman, 2001)

En el campo del diseño se registra un proceso similar: identidad y globalización ocupan una posición central en el discurso actual del diseño.

\footnotetext{
${ }^{6}$ ARCO LATINO, representado por los presidentes de la colectividad miembro, existe desde 1999 y se constituyó oficialmente como Asociación en junio de 2002 en Montpellier.

${ }^{7}$ Tendencias interculturales euro mediterráneas 2010 op.cit.

${ }^{8}$ Tendencias interculturales. 2010. op.cit.

${ }^{9}$ Tendencias interculturales. 2010. op.cit.
} 
Para Rigotti (2006) «la identidad y el Multiculturalismo son términos «imbuidos de malentendidos» en tanto que en la medida que se intentan definir, aumentarán las preguntas mientras las respuestas en gran parte quedan abiertas "El que espera trabajar con conceptos establecidos, tendrá que desechar tal suposición”.

Bonsiepe manifiesta desde su experiencia profesional en los países "de la periferia", su preocupación ante la insistencia con que surgen las siguientes preguntas: ¿existe un diseño típicamente brasileño, argentino o mexicano? En caso de haberlo ¿cómo se diferencia éste del diseño japonés, italiano o sueco? En otras palabras: ¿cuál es su identidad?, y dice:

"Antes de abordar estas preguntas -dice- conviene elaborar el mapa de los significados del término identidad con grupos estructurados de acuerdo con la proximidad semántica".

Distingue cinco grupos para construir una red semántica del concepto identidad. Esta lista puede crecer más, y no tiene ni centro ni jerarquía ${ }^{10}$.

\footnotetext{
${ }^{10}$ Bonsiepe, G. (2012) op., cit.
} 


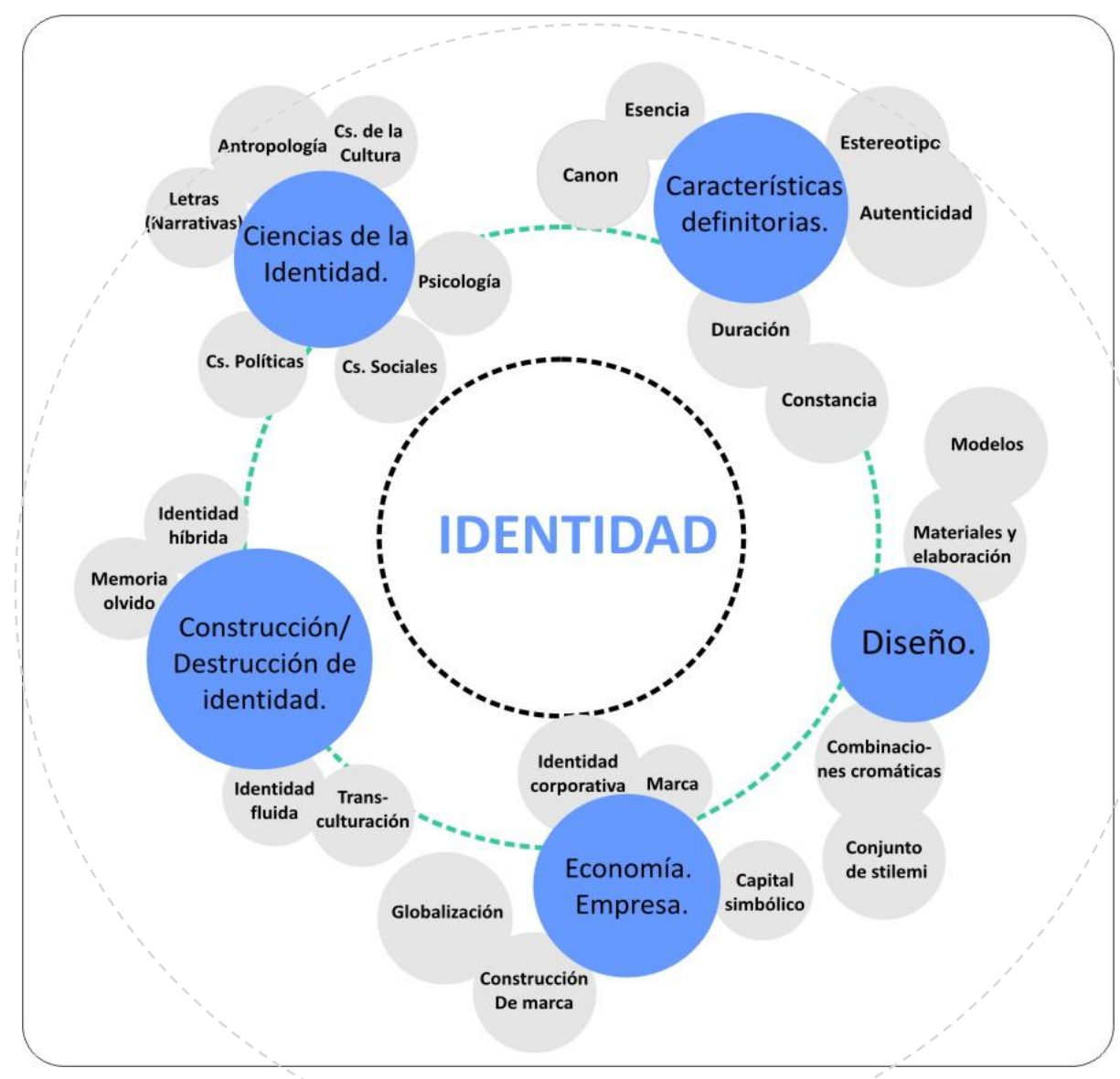

Fig. 5 Red semántica del concepto Identidad según criterios de G. Bonsiepe 2012.

Zygmunt Bauman, desde el campo de las ciencias sociales, también se alinea en contra de la idea de la identidad como algo fijo, duradero, cerrado, propio, esencial (Bauman 2007). « La identidad" se nos revela solo como algo que hay que inventar en lugar de descubrir». ${ }^{11}$ En otras palabras, las identidades no son esencias escondidas en algún lugar secreto, pero sí que son algo que debe ser creado - o, en la terminología del diseño, algo que debe ser diseñado-.

Dejando claro así que este concepto, va más allá de la construcción de marca o del diseño de la identidad corporativa.

\footnotetext{
${ }^{11}$ Bauman, Z. op. Cit. P.40.
} 
Juan Goytisolo hace la siguiente afirmación:

"La identidad no puede aceptarse como algo completo ni definitivo, muy al revés es una posibilidad siempre abierta [...] la verdadera identidad es una corriente continua, alimentada de infinidad de arroyos o cauces" (Goytisolo 1985)

Entendemos por lo tanto que el binomio diseño-identidad ha de ser el resultado de una búsqueda y análisis pero también producto de la acción proyectual, llegando más allá de la construcción de marca o del recurso publicitario, extrayendo aquellos elementos significativos que evolucionarán al ritmo de las sociedades.

Finalmente para captar la variedad de significados del concepto identidad en el campo del diseño puede ser útil una lista planteada por Bonsiepe que: "a pesar de no ser exhaustiva debe tomarse como punto de partida para analizar cómo se materializa la identidad del diseño":

1. En la forma de un conjunto de características formales o cromáticas.

2. En las taxonomías de productos, es decir, los tipos de productos característicos de una cultura (Cita el ejemplo de la calabaza para el mate que tiene su origen en la cultura Guaraní).

3. En el uso de materiales locales y sus métodos de fabricación correspondientes.

4. En el uso de un método proyectual específico (empatía con una determinada tradición arraigada en una región)

5. En la temática (necesidades) típica del contexto.

(Bonsiepe, 2012)

Mientras la identidad basada en las características morfológicas y cromáticas tiende a fijarse en la persona del diseñador -como individuo creador-, en el polo opuesto -el polo social- se encuentra la identidad que parte de las necesidades específicas de un contexto.

Según Bonsiepe," la identidad del diseño no es tanto el resultado de una búsqueda sino más bien el resultado de la acción proyectual, es decir hacer diseño en vez de buscar una identidad en si misma. (Bonsiepe, 2012). 


\section{Conclusiones}

La búsqueda realizada en el análisis bibliométrico evidencian la ausencia de referencias específicas sobre "Diseño Mediterráneo", la documentación y referencias existentes son muy diversas pero no abordan este tema en profundidad.

Surgen asociaciones a la terminología desde otras áreas, como la arquitectura, la moda, la decoración, incluso la gastronomía, pero desde puntos de vista parciales sin base científica o al menos consensuada por investigadores o teóricos.

Lo opuesto ocurre cuando realizamos la búsqueda sobre el Diseño Escandinavo o el Diseño italiano por ejemplo, surgiendo gran cantidad de autores, teóricos y bibliografía extensa para estos epígrafes.

La identidad regional está determinada por la identidad cultural y se puede definir como el sentimiento subjetivo del individuo y como tal no puede aceptarse como algo completo ni definitivo, muy por el contrario es una posibilidad siempre abierta.

La identidad cultural de una región es una corriente continua, alimentada por variedad de vertientes y como tal no depende tanto de lo que cada uno es o tiene, sino de aquello que pertenece al imaginario colectivo como artefactos de comunicación. Cómo nos ven los demás.

Se pueden identificar cinco grandes grupos de atributos que permiten construir una red semántica del concepto Identidad Mediterránea.

1. Conjunto de características formales o cromáticas

2. Tipos de productos característicos de una cultura

3. $\quad$ El uso de materiales locales y sus métodos de fabricación.

4. En el uso de un método proyectual específico.

5. En la temática (necesidades) típica del contexto. ${ }^{12}$

La Identidad Mediterránea aplicada al diseño no es tanto el resultado de una búsqueda sino más bien el resultado de la acción proyectual.

\footnotetext{
${ }^{12}$ Bonsiepe 2012, G. op, cit.
} 


\section{Bibliografía}

Bauman, Z. (2001). La identidad en un mundo globalizado, en La sociedad individualizada. Madrid: Catedra.

Bonsiepe, G. (2012). Diseño y crisis. Valencia: Campgráfic.

Calvera, A. (2010). “Cuestiones de fondo: la hipótesis de los tres orígenes del diseño”, En "Diseño e historia: tiempo, lugar y discurso", (Ed.) Isabel Campi, Oscar Salinas, Raquel Pelta, Anna Calvera, Guy Julier, Viviana Narotzky, Mireia Freixa and Concha Bayo. Barcelona: Designio.

Chaves, N. (2006). La imagen corporativa. Barcelona: Gustabo Gili.

Cooley, C. (1922). Human Nature and the Social Order. New York: Charles Scribner's Sons.

Gil, J. (2002). El nuevo diseño artesanal. Análisis y prospectiva en México. Barcelona: Universitat Politècnica de Catalunya, Departament de Proyecte d'Enginyeria.

Goytosolo, J. (1985) Contracorrientes, Barcelona: G.Gili.

Gonzalez, I. (1999). Conservación de bienes culturales: teoría, historia, principios y normas. Madrid: Ediciones Catedra.

Jaén, M., Martínez, F. (2002). El mediterráneo: confluencia de culturas. Paris: Presses Paris Sorbonne.

James, W. (1890). Principles of Psychology. Chicago: Enciclopedia Británica.

Julier, G. (2010). La cultura del diseño. Barcelona: Gistavo Gili. 113.

Julier, G. (2005). "Design and accumulation of cultural value” en "300\% Spanish Design". Barcelona: Electa.

Margolin, V., \& Buchanan, R. (1993). Descubriendo el diseño: el informe de un congreso. Temes de disseny, (9), 19-21.

Maldonado, T. (2015). Teoría de la conspiración. Bs. As.: Nueva Visión.

Merani, A. (1976). Historia crítica de la psicología. México: Grijalbo.

Molano, O, L. (2008). Identidad cultural: un concepto que evoluciona. Revista Opera, $\mathrm{N}^{\circ} 7$.

Poynor, Rick. (1998) Design beyond design: critical reflection and the practice of visual communication. Jan Van Eyck Akademie.

Raulik, G. C. (2008). National design strategies and country competitive economic advantage. The Design Journal, 11(2), 119-136.

Rae (Real academia española) (2010) Diccionario de la lengua española. Madrid: Espasa-Calpe. 
Diseño mediterráneo. Análisis preliminar de epígrafes.

Sparke, P. (2010). Diseño y cultura. Una introducción. Desde 1900 hasta la actualidad. Barcelona: GG Diseño.

Triana, N. R., López, M. L., \& Vásquez, J. J. C. (2012). Diseño y bienestar humano: puntos de encuentro a partir de metodologías de diseño. Iconofacto, 8(10).

Torregrosa, J.(1983). Perspectivas y contextos de la psicología social.Torregro Barcelona: Editorial Hispano Europea.

Young, Pauline V. (1939). Scientific social surveys and research: An introduction to the background, content, methods, and analysis of social studies. Prentice-Hall sociology series., (pp. 226-254). New York,Revista Objets,Vol.19. 